\title{
Mediastinal hematoma caused by subclavian catheterization
}

\author{
Hohyun Kim ${ }^{1,2,3}$, Chan Ik Park ${ }^{1,2}$, Dongyeon Ryu ${ }^{1,2}$, Kangho Lee ${ }^{1,2}$, \\ Jae Hun Kim $^{1,2,4}$ \\ ${ }^{1}$ Department of Trauma Surgery and Surgical Critical Care, ${ }^{2}$ Biomedical Research Institute, \\ Pusan National University Hospital, Busan, ${ }^{3}$ Department of Emergency Medicine, \\ ${ }^{4}$ Department of General Surgery, Pusan National University School of Medicine, Yangsan, \\ Korea
}

Received May 14, 2020

Revised June 8, 2020

Accepted June 11,2020

Correspondence to

Hohyun Kim

Department of Trauma Surgery and

Surgical Critical Care, Pusan National

University Hospital, Pusan National

University School of Medicine, 179

Gudeok-ro, Seo-gu, Busan 49241,

Korea

Tel: +82-51-240-7369

Fax: +82-51-240-7719

E-mail: traumagskhh@naver.com
Similar to all invasive procedures, central venous catheter placement is associated with a number of complications and strategies have been developed to minimize them. Complications due to the procedure may occur during insertion of the catheter (e.g., arterial puncture resulting in bleeding, pneumothorax, and cardiovascular side effects) and/or during maintenance of the line (e.g., infection, thrombosis, or other mechanical risks). Among these complications, mediastinal hematoma caused by central venous catheter placement is an unusual event. We reported the case of inadvertent mediastinal hematoma following central venous catheter insertion in a patient who suffered multiple traumas and presented the outcome of conservative management.

Key Words: Mediastinum, Hematomas, Central catheterization, Complications, Subclavian veins

\section{Introduction}

Percutaneous subclavian vein catheterization is one of the most common procedures performed in trauma patients. Usually, subclavian venipuncture is a successful and uncomplicated procedure, and reported complication rates range from $0.3 \%$ to $12 \%$, according to physician experience and the definition of complications (1-3). Some potential complications include failure to locate or cannulate the vein, puncture of the subclavian artery, misplacement of the catheter (defined as placement of the catheter tip in the contralateral subclavian vein or in either jugular vein), pneumothorax, mediastinal hematoma, hemothorax, and injury to adjacent nerves $(1,2)$.
In this paper, we present the case of a patient with a relatively rare complication of mediastinal hematoma caused by subclavian vein catheterization.

\section{Case presentation}

A 39-year-old woman with no significant past medical history suffered a high-speed road traffic accident. She was admitted to the trauma emergency room at our hospital and was assessed along the lines of the Advanced Trauma Life Support protocol.

The patient complained of left leg pain and right chest pain. The Glasgow Coma Scale score was 15 at presentation, and the vital signs were within reference limits.

(c)This is an Open Access article distributed under the terms of the Creative Commons Attribution Non-Commercial License (http://creativecommons.org/licenses/by-nc/4.0) which permits unrestricted noncommercial use, distribution, and reproduction in any medium, provided the original work is properly cited.

Copyright (c) 2020 Korean Association for Research, Procedures and Education on Trauma. All rights reserved. 
Abdominal computed tomography (CT) showed grade III liver injury of the right lobe, accompanied by a small amount of perihepatic fluid without evidence of active bleeding ("blush"). Triple-lumen central venous catheter placement through the right subclavian vein was planned to guide fluid therapy and transfusion. The catheter was inserted using the Seldinger technique and guided over a guide wire after dilating the tract with a dilator. However, the blood could not be aspirated through the catheter; thus, the procedure was abandoned. Subsequently, left subclavian vein catheterization was attempted twice but was unsuccessful.

Further, the patient underwent chest contrast-enhanced computed tomography (CECT) that showed luminal narrowing of the right subclavian vein with blushing of contrast into chest wall collaterals with heterogeneous lesions, which suggested right mediastinal hematoma with injury to the right subclavian vein (Fig. 1). Because the patient's
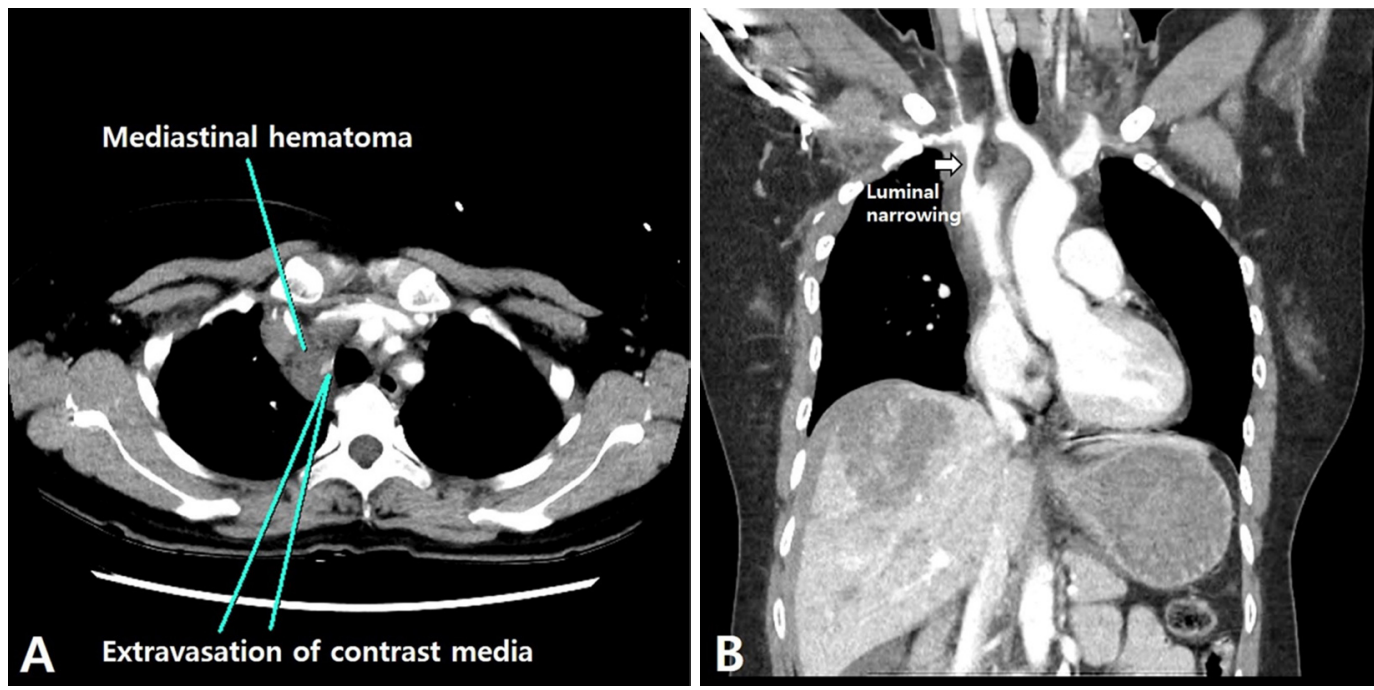

Fig. 1. Chest computed tomography (CT) scan shows a mediastinal hematoma with extravasation of contrast media in the right subclavian vein (A). Coronal view of the CT scan shows luminal narrowing of the right subclavian vein, indicating the presence of injury (B).
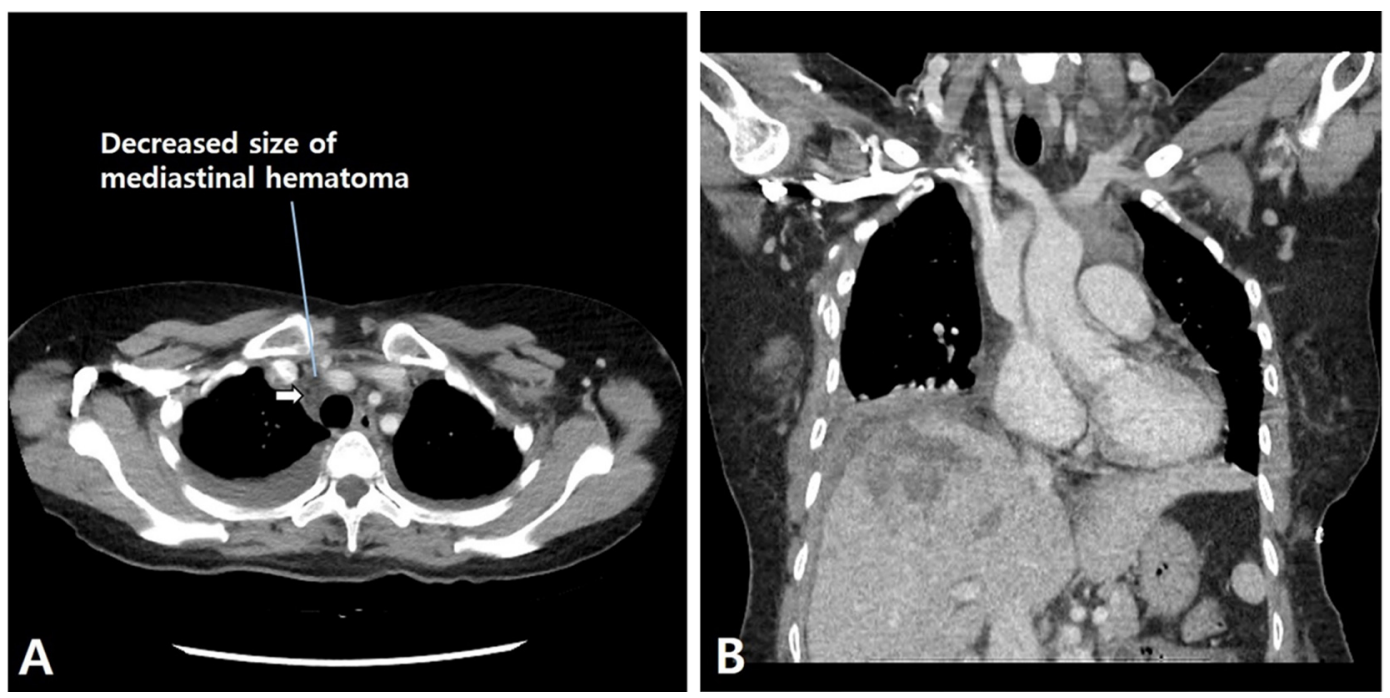

Fig. 2. Follow-up chest computed tomography (CT) scan on the seventh day after trauma shows no evidence of the extravasation of contrast media $(\mathrm{A}, \mathrm{B})$ and a reduction in the size of the mediastinal hematoma (A). 
vital signs were stable and she did not complain of chest tightness or dyspnea, conservative treatment with close observation was administered in the intensive care unit.

On the seventh day after trauma, follow-up chest CECT revealed no evidence of extravasation of contrast media and the size of the mediastinal hematoma had reduced (Fig. 2). Moreover, abdominal CT revealed a decrease in the amount of blood in the abdominal cavity with no active bleeding. Her stay at the hospital was uneventful, and she was discharged as per the protocol for discharge on the 14th day after trauma.

\section{Discussion}

Subclavian vein catheterization was introduced during the late 1970s and has become the preferred method of temporary vascular access. Indications for central catheter placement include inadequate peripheral venous access, peripherally incompatible infusions, hemodynamic monitoring, and extracorporeal therapies (1). However, acute complications, such as hemothorax, pneumothorax, right atrial perforation, air embolism, and delayed complications including stenosis and bacteremia associated with catheter placement have been consistently reported (1). Complication rates associated with subclavian vein catheterization range between $0.97 \%$ and $5.74 \%$, with pneumothorax being the most common complication $(1,2)$. Conversely, mediastinal hematoma secondary to subclavian vein catheterization is very rare.

Patients with mediastinal hematoma secondary to subclavian vein catheterization can be asymptomatic or present with symptoms such as dyspnea, low blood pressure, sweating, or orthopnea, depending on the size and location of the hematoma (3). Only one fatal case has been reported previously (4). Mediastinal hematoma should be considered in patients who undergo subclavian vein catheterization in the presence of persistent dyspnea and hypotension.

Even if a skilled and experienced physician performs subclavian vein catheterization, chest radiography should be performed to identify possible complications $(1,2)$. Thoracic CT is useful in case mediastinal hematoma is suspected (4). Follow-up chest radiography is sufficient to evaluate resorption if mediastinal hematoma occurs.
The mediastinal hematoma cases reported previously did not require any intervention to drain the hematoma and were all spontaneously resorbed, similar to that in our case $(3,5)$. We believe that as long as it does not impede vital functions, there is no need to drain the hematoma.

In conclusion, mediastinal hematoma may rarely complicate subclavian venous catheterization. Patients who develop complications may be asymptomatic or present with serious clinical manifestations that may be fatal. Cases with suspected mediastinal hematoma should be evaluated with thoracic $\mathrm{CT}$, and resorption can be monitored with chest CT.

\section{Conflict of interest}

No potential conflict of interest relevant to this article was reported.

\section{Acknowledgements}

This work was supported by a clinical research grant from Pusan National University Hospital in 2020.

\section{References}

1. Patel AR, Patel AR, Singh S, Singh S, Khawaja I. Central line catheters and associated complications: a review. Cureus. 2019;11(5):e4717.

2. Safety Committee of Japanese Society of A. Practical guide for safe central venous catheterization and management 2017. J Anesth. 2020;34(2):167-86.

3. Gupta P, Guleria S, Sharma S. Mediastinal haematoma: a rare complication following insertion of central venous catheter. Indian J Chest Dis Allied Sci. 2011; 53(4):225-8.

4. Arik N, Akpolat T, Demirkan F, Demirkazik F, Yasavul U, Turgan C, et al. Mediastinal hematoma - a rare complication of subclavian catheterization for hemodialysis. Nephron. 1993;63(3):354.

5. Yildirim F, Kara I, Yurumez B, Aygencel G, Turkoglu M. Asymptomatic mediastinal hematoma as a complication of ultrasound-guided internal jugular vein catheterization. Eur J Gen Med. 2016;13(1):85-6. 